\title{
Landauer Conductance of Luttinger Liquids with Leads
}

\author{
Dmitrii L. Maslov ${ }^{(a, b, c)}$ and Michael Stone ${ }^{(a)}$ \\ ${ }^{(a)}$ Department of Physics and ${ }^{(b)}$ Materials Research Laboratory \\ University of Illinois at Urbana-Champaign Urbana, IL 61801-3080, USA \\ ${ }^{(c)}$ Institute for Microelectronics Technology, Academy of Sciences of Russia, Chernogolovka, 142432 Russia
}

\begin{abstract}
We show that the dc conductance of a quantum wire containing a Luttinger liquid and attached to non-interacting leads is given by $e^{2} / h$ per spin orientation, regardless of the interactions in the wire. This explains the recent observations of the absence of conductance renormalization in long highmobility GaAs wires by Tarucha, Honda and Saku (Solid State Communications 94, 413 (1995)).

PACS numbers: 72.10.Bg, 73.20.Dx

Preprint number: P-95-05-023-i
\end{abstract}

For non-interacting electrons, the conductance of narrow ballistic quantum wires connected to wide reservoirs is quantized in units of $e^{2} / h$ [1].2]. When the effects of interactions are included this result is expected to be modified. In particular, when the electrons in the wire form a one-dimensional Luttinger liquid [3], the conductance is believed to be $K e^{2} / h$ per spin orientation [4] 6], where $K$ is the interaction dependent parameter characterizing the Luttinger liquid. For non-interacting electrons $K=1$. For repulsive interactions $K<1$, and the conductance should be reduced.

A recent experiment on very long $G a A s$ high mobility quantum wires [7] casts doubt on this picture. It is known that the same parameter $K$ enters the temperature dependence of the impurity correction to the conductance, and using this the authors of [7] where able to estimate $K$ to be about 0.7 for the electron gas in their wires, implying a conductance reduction of $30 \%$ in the ballistic limit. The actual reductions observed are only a few percent of $e^{2} / h$ however.

This observation leads us to re-assess the conventional analysis of the charge transport in Luttinger wires. In this paper we will argue that the conductance of a quantum wire attached to one-dimensional non-interacting leads (which are intended to model the higher-dimensional Fermi-liquid reservoirs) is $e^{2} / h$ regardless of the interactions in the wire itself. This is because the finite resistance of a ballistic wire is a contact resistance [8 11] and comes entirely from processes that take place outside the wire, when the electrons are not in the Luttinger-liquid state (cf. Fig. 11a). When the Umklapp scattering is negligible (which is the case for the low-density electron gas in semiconductor heterostructures), the interactions in the wire conserve the total momentum and thus do not change the resistance.

While it is generally asserted in the literature that $g=K e^{2} / h$ is the correct result for interacting electrons [4] (a) (at least for wires longer than the Fermi wavelength), we must remark there have been previous comments supporting the result $g=e^{2} / h$. In their earlier paper [12], Fisher and Kane remark that the ac conductance will cross over to the non-interacting value at frequencies lower than $\omega=v_{F} / L$, where $v_{F}$ is the Fermi velocity and $L$ is the wire length. Matveev and Glazman 13. make a similar remark when discussing the conductance of multi-mode interacting wires [14.

In this paper we will first calculate the conductance for a wire containing a homogeneous Luttinger liquid with parameter $K_{W}$ attached to leads which are also homogeneous but have parameter $K_{L}$. We will see that the conductance is given by $K_{L} e^{2} / h$. We will then prove a general theorem showing that even when $K$ and the density-wave velocity $v$ vary arbitrarily in the Luttinger liquid, the conductance is determined by the asymptotic value of $K$ only. As we assume that the electrons do not interact in the leads, this implies that $K_{L}=1$ and the conductance is given by $e^{2} / h$.

\section{KUBO FORMULA CALCULATION}

We start with the simple case of an infinite Luttinger liquid (LL) which contains three regions: the wire $(0 \leq x \leq L)$ and two leads $(x<0$ and $x>L$, respectively). The interaction parameter is assumed to change abruptly from $K_{L}$ in the leads to $K_{W}$ in the wire (Fig. [ $\mathrm{b}$ ). As the one-dimensional leads are supposed to model two- or three- dimensional Fermi-liquid leads, we will put $K_{L}=1$ at the end of the calculation. In a real system with wide leads, the applied electrostatic potential difference $V_{-}-V_{+}$produces an inhomogeneous electric field $E(x)$ which is concentrated in the wire and decays rapidly towards the open ends of the system. In the one-dimensional model we imitate this behavior by assuming that the field is zero outside the wire. Thus the current $I=e j$ is related to the field by

$$
I(x, t)=\int_{0}^{L} d x^{\prime} \int \frac{d \omega}{2 \pi} e^{-i \omega t} \sigma_{\omega}\left(x, x^{\prime}\right) \bar{E}_{\omega}\left(x^{\prime}\right),
$$


where $\bar{E}_{\omega}(x)$ is the time Fourier component of the electric field and $\sigma_{\omega}\left(x, x^{\prime}\right)$ is the non-local ac conductivity. In the Matsubara representation, $\sigma_{\omega}\left(x, x^{\prime}\right)$ is expressed via the (imaginary time) current-current correlation function by the usual Kubo formula

$$
\sigma_{\omega}\left(x, x^{\prime}\right)=-\frac{e^{2}}{\pi \bar{\omega}} \int_{0}^{\beta}\left\langle T_{\tau}^{*} j(x, \tau) j\left(x^{\prime}, 0\right)\right\rangle e^{-i \bar{\omega} \tau},
$$

where $t=i \tau$ and $\bar{\omega}$ is defined by $\omega=i \bar{\omega}+\epsilon$. The (Euclidean) action of the spinless Luttinger liquid is

$$
S_{E}=\frac{1}{8 \pi} \int d x \int_{0}^{\beta} d \tau \frac{1}{K(x)}\left\{\frac{1}{v(x)}\left(\partial_{\tau} \phi\right)^{2}+v(x)\left(\partial_{x} \phi\right)^{2}\right\} .
$$

In the bosonized form, the particle-number current is $j=$ $i \partial_{\tau} \phi / 2 \pi$, and Eq. (2) reduces to [15]

$$
\sigma_{\omega}\left(x, x^{\prime}\right)=-e^{2} \frac{\bar{\omega}}{\pi} G_{\bar{\omega}}\left(x, x^{\prime}\right)
$$

where

$$
G_{\bar{\omega}}\left(x, x^{\prime}\right)=\int_{0}^{\beta} \frac{d \tau}{(2 \pi)^{2}}\left\langle T_{\tau}^{*} \phi(x, \tau) \phi\left(x^{\prime}, 0\right)\right\rangle e^{-i \bar{\omega} \tau}
$$

is the propagator of the bosonic field. According to Eq. (11), we need to know $G_{\bar{\omega}}\left(x, x^{\prime}\right)$ only for $0 \leq x^{\prime} \leq L$. The propagator $G_{\bar{\omega}}\left(x, x^{\prime}\right)$ satisfies the equation

$$
\left\{-\partial_{x}\left(\frac{1}{K(x)} \partial_{x}\right)+\bar{\omega}^{2}\right\} G_{\bar{\omega}}\left(x, x^{\prime}\right)=\delta\left(x-x^{\prime}\right),
$$

which leads to the following boundary conditions: i) $G_{\bar{\omega}}\left(x, x^{\prime}\right)$ is continuous at $x=0, L$ and $x=x^{\prime}$, ii) $\frac{1}{K(x)} \partial_{x} G_{\bar{\omega}}\left(x, x^{\prime}\right)$ is continuous at $x=0, L$ but iii) undergoes a jump of unit height at $x=x^{\prime}$, i.e.,

$$
-\left.\frac{1}{K(x)} \partial_{x} G_{\bar{\omega}}\left(x, x^{\prime}\right)\right|_{x=x^{\prime}-0} ^{x=x^{\prime}+0}=1
$$

In addition, we assume that the infinitesimal dissipation is present in the leads, so that $G_{\bar{\omega}}\left( \pm \infty, x^{\prime}\right)=0$. (In a real- time formulation this corresponds to outgoing wave boundary conditions.) The solution to the problem defined above can be written in the form

$$
G_{\bar{\omega}}\left(x, x^{\prime}\right)= \begin{cases}A e^{|\bar{\omega}| x} & \text { for } x \leq 0 \\ B e^{|\bar{\omega}| x}+C e^{-|\bar{\omega}| x} & \text { for } 0<x \leq x^{\prime} \\ D e^{|\bar{\omega}| x}+E e^{-|\bar{\omega}| x} & \text { for } x^{\prime}<x \leq L \\ F e^{-|\bar{\omega}| x} & \text { for } x>L\end{cases}
$$

where $A \ldots F$ depend on $x^{\prime}$ and $\bar{\omega}$ and are to be found from the boundary conditions. As we are interested in the dc limit of $\sigma_{\omega}\left(x, x^{\prime}\right)$, the frequency $\bar{\omega}$ can be put to zero in all boundary conditions except for in Eq. (7). Consequently, $G_{\bar{\omega}}\left(x, x^{\prime}\right)$ becomes $x$ - and $x^{\prime}$-independent in this limit and is readily found to be equal to $-K_{L} / 2 \bar{\omega}$ in all regions. Thus the dc limit of the non-local conductivity is

$$
\lim _{\bar{\omega} \rightarrow 0+} \sigma_{\omega}\left(x, x^{\prime}\right)=\frac{K_{L} e^{2}}{2 \pi} .
$$

For a static electric field, $\bar{E}_{\omega}(x)=2 \pi \delta(\omega) E(x)$, and Eq. (11) gives the $x$ - and $t$-independent current

$$
I=\frac{K_{L} e^{2}}{2 \pi} \int_{0}^{L} d x^{\prime} E\left(x^{\prime}\right)=\frac{K_{L} e^{2}}{2 \pi}\left(V_{-}-V_{+}\right),
$$

from which we see that the conductance is

$$
g \equiv \frac{I}{V}=K_{L} \frac{e^{2}}{2 \pi} .
$$

We have been using units where $\hbar=1$ or $h=2 \pi$ so, restoring $\hbar$, we have $g=K_{L} e^{2} / h$. Thus the conductance is determined by the value of $K_{L}$ in the leads and does not depend on the value of $K_{W}$ in the wire.

Notice that in this calculation we have implicitly taken the limit $\omega \rightarrow 0$ before the $q \rightarrow 0$ limit $[16$ 18. The traditional order of limits is $q \rightarrow 0$ before $\omega \rightarrow 0$. The latter yields the Drude formula, which has a divergent dc limit for perfect systems. The former produces a finite Landauer dc conductance even for a perfect system. It furthermore corresponds to the experimental situation where a static field is applied over a finite region.

From our assumption of Fermi-liquid leads we have $K_{L}=1$, consequently $g=e^{2} / h$. This result is independent of the wire length — at least until the wire is so long that impurity scattering becomes significant. Similar calculations can be done for two Luttinger-liquid wires connected in series and separated by the Fermi-liquid region. Again, the dc conductance is found to be independent of the interactions in the wires and is given by $e^{2} / h$, even in the case when these interactions are of different strengths.

\section{CONDUCTANCE THEOREM}

We can get some further insight into the result of the previous section, and establish a general theorem, by considering a generic inhomogeneous system and asking how it responds when an electric field is switched on at some particular moment.

Let $\Omega$ be a finite segment of the wire and assume that the Luttinger liquid has values of $K$ and $v$ that vary smoothly and arbitrarily within $\Omega$ but take constant values $K_{L}, v_{L}$ in the leads outside $\Omega$.

The real time effective action for a spinless Luttinger liquid is

$$
S=\frac{1}{8 \pi} \int d^{2} x \frac{1}{K(x)}\left\{v(x)\left(\partial_{x} \phi\right)^{2}-\frac{1}{v(x)}\left(\partial_{t} \phi\right)^{2}\right\} .
$$

We have chosen to normalize the $\phi$ field so that 


$$
\rho=-\frac{1}{2 \pi} \partial_{x} \phi, \quad j=\frac{1}{2 \pi} \partial_{t} \phi .
$$

The interaction with an external electromagnetic field $A_{\mu}$ is given by

$$
S_{i n t}=\frac{e}{2 \pi} \int d^{2} x\left\{-A_{0} \partial_{x} \phi+A_{1} \partial_{t} \phi\right\},
$$

so the equation of motion for the field (classical or quantum) is

$$
\frac{1}{4 \pi}\left[\partial_{t}\left(\frac{1}{K v} \partial_{t} \phi\right)-\partial_{x}\left(\frac{v}{K} \partial_{x} \phi\right)\right]=\frac{e}{2 \pi} E(x, t),
$$

where $E=\partial_{x} A_{0}-\partial_{t} A_{1}$ is the electric field.

We seek a solution to this equation when the electric field (which we will assume to be non-zero only in the region $\Omega$ ) is switched on in some manner at time $t=0$ and remains constant (but not necessarily homogeneous) thereafter. We will select a particular transient time dependence for the field in a few lines, but remark here that if the conductance is to be well-defined then it must be insensitive to this choice.

We find the solution by first solving the time independent problem

$$
-\frac{1}{2} \partial_{x}\left(\frac{v(x)}{K(x)} \partial_{x} \phi\right)=e E(x) .
$$

Call the solution to this problem $\Phi(x)$. For example if $v=K=1$ and $E(x)=E, x \in[0, L],=0$ otherwise, then

$$
\Phi(x)=\left\{\begin{array}{ll}
e E L x & \text { for } x<L ; \\
-e E x(x-L) & \text { for } x \in[0, L] ; . \\
-e E L(x-L) & \text { for } x>L
\end{array} .\right.
$$

In general, being the solution of a second-order equation, the function $\Phi$ contains two constants of integration. One is simply an additive constant and the other will be chosen to ensure, as we have done in Eq. (17), that the outer, linear, portions of the solution have equal and opposite slopes. With this choice it should now be clear that

$$
\phi(x, t)=\max (0, \Phi(x)+\alpha t)
$$

will solve

$$
\frac{1}{4 \pi}\left[\partial_{t}\left(\frac{1}{K v} \partial_{t} \phi\right)-\partial_{x}\left(\frac{v}{K} \partial_{x} \phi\right)\right]=\frac{e}{2 \pi} E(x)
$$

at late enough time, provided $\alpha$ is chosen so that the points of intersection of $\Phi(x)+\alpha t$ with the $x$ axis move out in the $\pm x$ directions at velocity $\pm v_{L}$. The time must be late enough that there are only two such intersections (we will call the one on the left $A(t)$ and on the right $B(t))$ and they take place outside $\Omega$. The two points of intersection will move at the same speed but in opposite directions because of the opposite slopes of the linear portions of the solution. We may find $\alpha$ by integrating
Eq. (19) over an interval $[a, b]$ containing $\Omega$, but itself contained in $[A(t), B(t)]$. We find

$$
\frac{1}{2}\left[\frac{v_{L}}{K_{L}} \partial_{x} \phi\right]_{a}^{b}=\int_{a}^{b} e E d x .
$$

The magnitude of the slopes are therefore

$$
\left|\partial_{x} \phi\right|_{a, b}=\frac{K_{L}}{v_{L}} \int_{a}^{b} e E d x .
$$

The requirement that the linear portions of $\phi(x, t)$ move out at speed $v_{L}$ then gives

$$
\alpha=\partial_{t} \phi=\left.v_{L} \partial_{x} \phi\right|_{b}=K_{L} \int_{a}^{b} e E d x .
$$

We can make this solution valid at all times by switching on the field in the manner given by substituting Eq. (18) in Eq. (15). This is the particular transient behavior for $E$ alluded above. If we do not switch the field on in this way then there will be a region at the head of each outgoing wave where the solution will be different from Eq. (18), but this transient behavior will not affect the dc conductance.

Since the electric current $I$ is given by $I=e j=$ $-e \partial_{t} \phi / 2 \pi$ our solution has

$$
I(x)= \begin{cases}\frac{e^{2}}{2 \pi} K_{L} \int E\left(x^{\prime}\right) d x^{\prime} & \text { for } x \in[A(t), B(t)] \\ =0 & \text { elsewhere }\end{cases}
$$

As before, we have been working with units where $\hbar=1$, so again restoring $h$ in Eq. (23) gives

$$
I=\frac{e^{2}}{h} K_{L}\left(V_{-}-V_{+}\right)
$$

Physically what is occuring is that once the electric field is switched on there are different equilibrium densities on the left and right sides of $\Omega$, since these regions are at different potentials $V_{+,-}$. The extra charge $\delta \rho=-\partial_{x} \phi / 2 \pi= \pm\left(e K_{L} / \pi v_{L}\right) \delta V$ flows into and out of the "reservoirs" or asymptotic leads in the form of a "shock wave" whose head moves at $\pm v_{L}$. This flow of charge provides the current in the leads which is obviously equal to $I=\delta \rho v_{L}$. The current is therefore determined only by the asymptotic values of $v$ and $K$. As we said above, different ways of switching on the field will lead to transients at the head of the wave of charge, but will not alter the current following the head.

The analysis of this and previous sections can readily be generalized for the case of electrons with spin. In this case, the conductance per spin orientation is obtained from Eqs. (9.24) by replacing $K_{L} \rightarrow K_{L}^{\rho}$, where $K_{L}^{\rho}=1$ parameterizes the charge part of the Luttinger liquid in the leads. 


\section{DISCUSSION}

We have shown in Secs. II, II that the conductance of a ballistic wire containing Luttinger liquid and connected to non-interacting leads is not renormalized by the interactions in the wire. It remains at the non-interacting value $g=e^{2} / h$ per spin orientation.

We now discuss some experimental implications of this result. At low temperatures $(T<1.2 \mathrm{~K})$, the temperature dependence of the conductance of quasi-ballistic GaAs wires appears [7 to be described reasonably well by the theory of disordered Luttinger liquids [4,6]. The value of the $K$-parameter extracted from the exponent of this dependence is $\approx 0.7[7]$. This suggests that at higher temperatures, when the disorder-induced reduction of the conductance is not pronounced yet, the conductance should be $0.7 e^{2} / h$, i.e., significantly smaller than the conductance quantum. The observed higher-temperature value of the conductance is very close to $e^{2} / h$ however, whereas the total change in the conductance in the whole range of temperatures is $1-5 \%$, depending on the length of the wire. Although we have considered only the case of a pure wire in this paper, it would be reasonable to expect that reduction in the conductance due to disorder in the wire would depend on the $K$-parameter of the wire. We thus can conclude that the two experimental results-the Luttinger-liquid-like temperature dependence at lower temperatures and absence of the conductance renormalization at higher temperatures-do not contradict to each other.

Finally, we note that the transport in quantum wires in zero magnetic fields is very different from the edgestate transport in the fractional quantum Hall effect system [19,20]. In the former case, electrons in the wide leads are necessarily not in the Luttinger-liquid state and the finite conductance $e^{2} / h$ arises from the scattering of Fermi-liquid electrons at the contacts. In the latter case, strong magnetic field binds electrons to the edges even in the leads (at least in the 2DEG parts of the leads adjacent to the constriction), thus both incoming and outgoing electrons are in the Luttinger-liquid state, with the parameter $K$ given by the filling fraction $\nu$. In this case one expects to have both the conductance renormalization in the absence of tunneling between edges $\left(g=\nu e^{2} / h\right)$ and the temperature-dependent tunneling rate.

Note Added: While we were in the process of writing this paper we learned of a recent preprint by Safi and Schulz (cond- mat/9505079) who also conclude that the conductance is not renormalized by the interactions in the wire.

\section{ACKNOWLEDGMENTS}

This work was supported by the NSF under grants DMR94-24511 and DMR89-20538. DLM would like to thank Y. B. Levinson for discussions over many years of the physics behind the Landauer formula. We both thank D. Loss for useful conversations and drawing our attention to the recent work of Safi and Schulz, as well as to Ref. 18]. We also thank P. M. Goldbart for his persistent encouragement.

[1] B. J. van Wees et al., Marel and Phys. Rev. Lett. 60, 848 (1988).

[2] D. A. Wharam et al., A. C. Jones, J. Phys. C 21, L209 (1988).

[3] F. D. M. Haldane, J. Phys. C 14, 2585 (1981).

[4] W. Apel and T. M. Rice, Phys. Rev. B 26, 7063 (1982).

[5] C. L. Kane and M. P. A. Fisher, Phys. Rev. B 46, 15233 (1992).

[6] M. Ogata and H. Fukuyama, Phys. Rev. Lett. 73, 468 (1994).

[7] S. Tarucha, T. Honda, T. Saku, Sol. State Commun. 94, 413 (1995).

[8] Y. Imry, in Directions in Condensed Matter Physics, edited by G. Grinstein and G. Mazenko (World Scientific, Singapore, 1986), Vol. 1, p. 101.

[9] R. Landauer, Z. Phys. B 68, 217 (1987).

[10] L. I. Glazman, G. B. Lesovik, D. E. Khmel'nitskii and R. I. Shekhter, Pis'ma Zh. Eksp. Teor. Fiz. 48, 218 (1988) [Sov. Phys. JETP. Lett. 48, 238 (1988)].

[11] Y. B. Levinson, Zh. Eksp. Teor. Fiz. 95, 2175 (1989) [Sov. Phys. JETP. 68, 1257 (1989)].

[12] C. L. Kane and M. P. A. Fisher, Phys. Rev. Lett. 68, 1220 (1992).

[13] K. A. Matveev and L. I. Glazman, Physica B 71, 266 (1993).

[14] DLM is grateful to K. A. Matveev for pointing this out for him in a private conversation.

[15] R. Shankar, Int. J. of Mod. Phys. B 4, 2371 (1990).

[16] D. S. Fisher and P. A. Lee, Phys. Rev. B 23, 6851 (1981).

[17] E. Fenton, Phys. Rev. B 46, 3754 (1992).

[18] H. J. Schulz, 1994 Les Houches lecture notes condmat/9503150).

[19] F. P. Milliken, C. P. Umbach and R. A. Webb, preprint (1994).

[20] K. Moon, C. L. Kane. S. M. Girvin and M. P. A. Fisher, Phys. Rev. Lett. 71, 4381 (1993).

FIG. 1. (a) The quantum wire containing Luttinger liquid (LL) and connected to Fermi-liquid (FL) leads. Finite resistance arises from the scattering of FL electrons at the contacts. (b) Effective 1D model for the situation depicted in (a). 


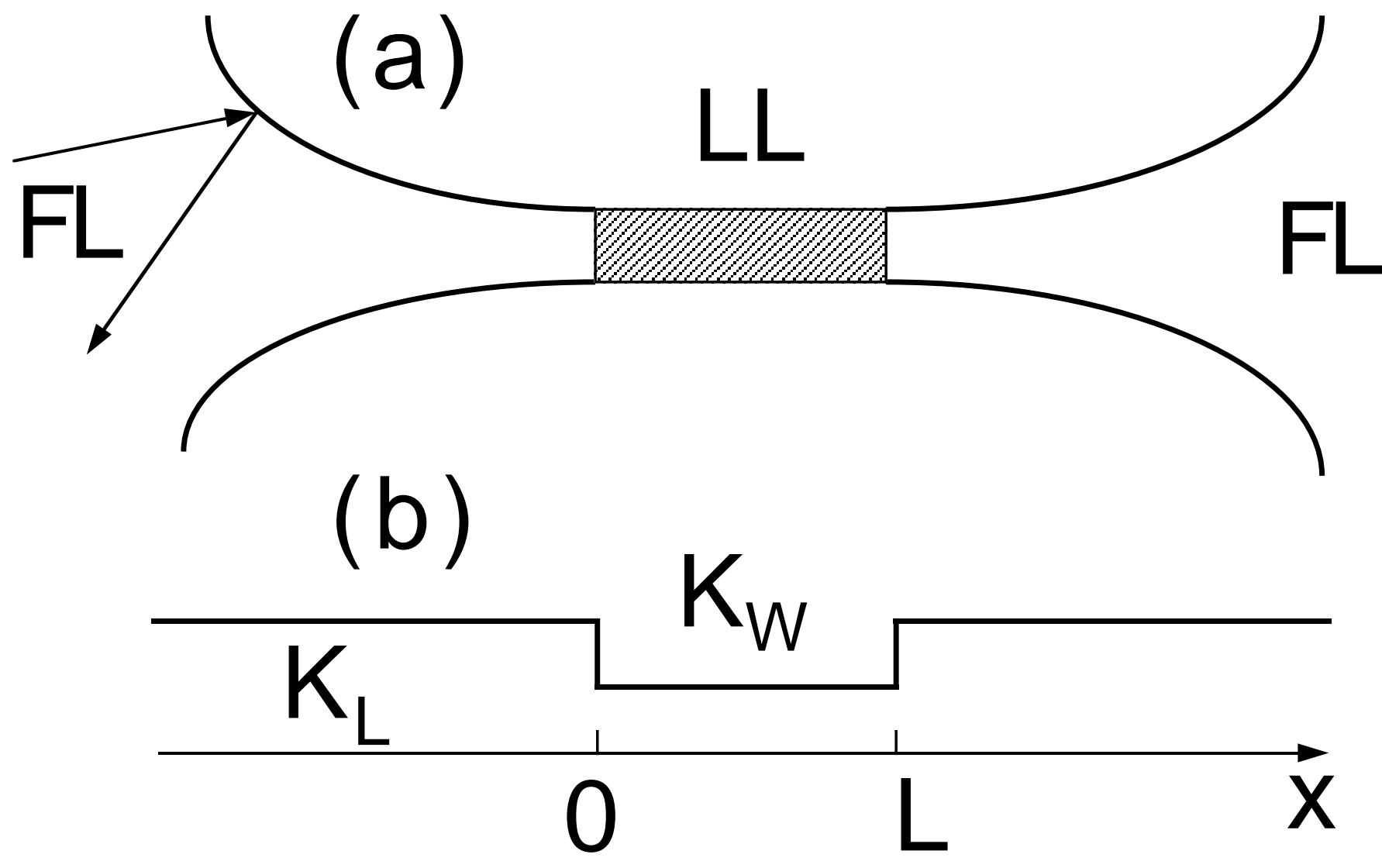

